\title{
Which time of the day do we have the lowest hydration status?
}

\author{
A. Athanasatou, O. Malisova and M. Kapsokefalou \\ Unit of Human Nutrition, Department of Food Science and Technology, Agricultural University of Athens, Athens, \\ Greece
}

Mild dehydration may occur during the day because of poor drinking and eating habits or poor accessibility to a variety of fluids or foods ${ }^{(1)}$. Data on simultaneous water intake and hydration status from healthy free-living subjects are limited. The evaluation of hydration indices measured in every individual urine sample collected throughout a day may reveal potential intervals of mild dehydration and subsequently encourage focused advice on hydration schemes ${ }^{(2)}$. It is therefore of unequivocal importance to measure a) urine hydration indices in short time interval $(6 \mathrm{~h})$ urine samples, collected over a $24 \mathrm{~h}$ period and b) water intake from all sources per time interval $(6 \mathrm{~h})$. Linking information on hydration status, water intake and variety of beverages intake in short time intervals during the day will elucidate factors that may lead to mild dehydration.

A total of 164 healthy subjects (aged $38 \pm 12 \mathrm{yrs}$; BMI $24.9 \pm 4.7 \mathrm{~kg} / \mathrm{m}^{2}$ ) were enrolled in the study, which was conducted in Athens, Greece (6-8/2013, 1-2/2014, 6-7/2014). Subjects recorded their food and drink intakes and recorded and collected all urine specimens for three consecutive days. Water intake and urine hydration indices were assessed in $6 \mathrm{~h}$ intervals over the day from wake up time. Hydration indices (volume, osmolality, urine specific gravity (USG) and colour) were measured in all $6 \mathrm{~h}$ intervals, first morning and $24 \mathrm{~h}$ urine samples. Beverages consumption consisted of the following categories: 1) hot beverages; 2) milk; 3) fruit and vegetable juices; 4) caloric soft drinks; 5) diet soft drinks; 6) alcoholic drinks; 7) water and 8) other beverages.

Values of hydration indices of morning, afternoon, evening, overnight, first morning and $24 \mathrm{~h}$ urine samples.

\begin{tabular}{|c|c|c|c|c|c|c|c|c|c|c|c|c|}
\hline \multirow{2}{*}{ Hydration Indices } & \multicolumn{2}{|c|}{ Morning } & \multicolumn{2}{|c|}{ Afternoon } & \multicolumn{2}{|c|}{ Evening } & \multicolumn{2}{|c|}{ Overnight } & \multicolumn{2}{|c|}{$\begin{array}{l}\text { First morning } \\
\text { urine }\end{array}$} & \multicolumn{2}{|l|}{$24 \mathrm{~h}$} \\
\hline & Mean & SD & Mean & SD & Mean & SD & Mean & SD & Mean & SD & Mean & SD \\
\hline Volume (mL) & 557 & 231 & 378 & 205 & 290 & 158 & 177 & 149 & 339 & 123 & 1331 & 144 \\
\hline $\begin{array}{l}\text { Osmolality } \\
\text { (mOsm/kg) }\end{array}$ & 620 & 240 & 627 & 258 & 580 & 254 & 271 & 157 & 691 & 224 & 665 & 223 \\
\hline USG & $1 \cdot 017$ & $0 \cdot 006$ & $1 \cdot 017$ & 0.006 & 1.019 & $0 \cdot 007$ & $1 \cdot 016$ & $0 \cdot 007$ & 1.018 & 0.008 & 1.017 & 0.006 \\
\hline Colour & 3 & 2 & 3 & 1 & 3 & 2 & 1 & 2 & 3 & 1 & 4 & 2 \\
\hline
\end{tabular}

The effect of time was significant for volume $(F=117 \cdot 191 ; p<0 \cdot 001)$, osmolality $(F=65 \cdot 228 ; p<0 \cdot 001), U S G(F=5 \cdot 096 ; p=$ $0.003)$ and colour $(\mathrm{F}=65.123 ; \mathrm{p}<0.001)$. Urine osmolality measured in the morning interval and first morning urine samples were far more likely to accurately reflect $24 \mathrm{~h}$ urine osmolality, compared to evening, afternoon and overnight intervals (Linear Regression Models). The urine volume of the morning interval samples reflects by $76 \%$ the urine excretion over a day ( $24 \mathrm{~h}$ collection).

Mean daily total water intake was $2266 \mathrm{~mL}$ (SD 781). For water, the most popular beverage consumed, mean daily intake was 1168 $\mathrm{mL}$ (SD 666); following hot beverages $376 \mathrm{~mL}$ (SD 245), alcoholic drinks $198 \mathrm{~mL}$ (SD 237), caloric soft drinks $188 \mathrm{~mL}$ (SD 120), milk $160 \mathrm{~mL}$ (SD 123), fruit and vegetable juice $147 \mathrm{~mL}$ (SD 101). Total beverages intake was positively correlated with total daily water intake $(r=0.896 ; p<0.001)$ and variety score $(r=0.238 ; p<0.001)$. A peak on beverage intake was observed in the morning (hot beverages: $211 \mathrm{~mL}$ (SD 120); milk: $133 \mathrm{~mL}$ (SD 104)) and in the evening (alcoholic drinks: $154 \mathrm{~mL}$ (SD138)). Water was preferred to other type of beverages throughout all short time intervals of day.

In conclusion, the produced urine volume was greater in the morning interval and decreased throughout the day. This fluctuation in urine volume follows the fluctuation of water intake by time. A morning interval sample, and not a spot urine sample (e.g. first morning urine sample) may be able to replace $24 \mathrm{~h}$ urine collection.

This study was a Student Research Grant supported by EHI.

1. Lemaire JB, Wallace JE, et al. BMC Health Serv Res. 2010; 10:241

2. Perrier E, Demazieres A, et al. Eur J Appl Physiol. 2013; 113:2143-2151 